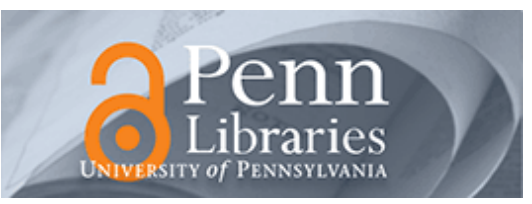

University of Pennsylvania

ScholarlyCommons

January 2006

\title{
Physical Insight Into the "Growing" Evanescent Fields of Double- Negative Metamaterial Lenses Using Their Circuit Equivalence
}

\author{
Andrea Alù \\ University of Pennsylvania, andreaal@seas.upenn.edu \\ Nader Engheta \\ University of Pennsylvania, engheta@ee.upenn.edu
}

Follow this and additional works at: https://repository.upenn.edu/ese_papers

\section{Recommended Citation}

Andrea Alù and Nader Engheta, "Physical Insight Into the "Growing" Evanescent Fields of Double-Negative Metamaterial Lenses Using Their Circuit Equivalence", . January 2006.

NOTE: At the time of publication, author Andrea Alù was affiliated with the University of Roma Tre. As of September 2006, she is a staff member in the Department of Electrical and Systems Engineering at the University of Pennsylvania. Copyright 2006 IEEE. Reprinted from IEEE Transactions on Antennas and Propagation, Volume 54, Issue 1, January 2006, pages 268-272.

This material is posted here with permission of the IEEE. Such permission of the IEEE does not in any way imply IEEE endorsement of any of the University of Pennsylvania's products or services. Internal or personal use of this material is permitted. However, permission to reprint/republish this material for advertising or promotional purposes or for creating new collective works for resale or redistribution must be obtained from the IEEE by writing to pubs-permissions@ieee.org. By choosing to view this document, you agree to all provisions of the copyright laws protecting it.

This paper is posted at ScholarlyCommons. https://repository.upenn.edu/ese_papers/175

For more information, please contact repository@pobox.upenn.edu. 


\title{
Physical Insight Into the "Growing" Evanescent Fields of Double-Negative Metamaterial Lenses Using Their Circuit Equivalence
}

\begin{abstract}
Pendry in his paper, "Negative refraction makes a perfect lens" (Phys. Rev. Lett., vol. 85, no. 18, pp. $3966-3969,2000$ ) put forward an idea for a lens made of a lossless metamaterial slab with $n=-1$, that may provide focusing with resolution beyond the conventional limit. In his analysis, the evanescent wave inside such a lossless double-negative (DNG) slab is "growing," and thus it "compensates" the decaying exponential outside of it, providing the subwavelength lensing properties of this system. Here, we examine this debated issue of "growing exponential" from an equivalent circuit viewpoint by analyzing a set of distributed-circuit elements representing evanescent wave interaction with a lossless slab of DNG medium. Our analysis shows that, under certain conditions, the current in series elements and the voltage at the element nodes may attain the dominant increasing due to the suitable resonance of the lossless circuit, providing an alternative physical explanation for "growing exponential" in Pendry's lens and similar subwavelength imaging systems.
\end{abstract}

\section{Keywords}

Double-negative (DNG) metamaterials, left-handed (LH) metamaterials, subwavelength resolution.

\section{Comments}

NOTE: At the time of publication, author Andrea Alù was affiliated with the University of Roma Tre. As of September 2006, she is a staff member in the Department of Electrical and Systems Engineering at the University of Pennsylvania. Copyright 2006 IEEE. Reprinted from IEEE Transactions on Antennas and Propagation, Volume 54, Issue 1, January 2006, pages 268-272.

This material is posted here with permission of the IEEE. Such permission of the IEEE does not in any way imply IEEE endorsement of any of the University of Pennsylvania's products or services. Internal or personal use of this material is permitted. However, permission to reprint/republish this material for advertising or promotional purposes or for creating new collective works for resale or redistribution must be obtained from the IEEE by writing to pubs-permissions@ieee.org. By choosing to view this document, you agree to all provisions of the copyright laws protecting it. 


\section{CONCLUSION}

The preconditioning scheme adopting the interactions between elements inside a cell of the array —SSRs or thin-strips - as blocks excels as a suitable tool to analyze systematically and most efficiently finite composite structures in metamaterials. It has been compared with traditionally successful tools in the MoM-EFIE analysis such as the ILU preconditioner relying on a geometrically based selection of a bandeddiagonal portion of $Z$, for the cases where the required resources are available in our PC, and a blockwise memory-efficient modification for the case of problems with very large number of unknowns. In all the cases tested, the geometric block-diagonal preconditioner reaches convergence in less number of iterations and total computational time.

\section{REFERENCES}

[1] V. G. Veselago, "The electrodynamics of substances with simultaneously negative values of $\varepsilon$ and $\mu$," Sov. Phys. Usp., vol. 10, no. 509, 1968.

[2] C. C. Chen, "Scattering by a two-dimensional periodic array of conducting plates," IEEE Trans. Antennas Propag., vol. AP-18, pp. 660-665, Sep. 1970.

[3] Y. Saad, Iterative Methods for Sparse Linear Systems. Boston, MA: PWS, 1996.

[4] K. Forsman, W. Gropp, L. Kettunen, D. Levine, and J. Salonen, "Solution of dense systems of linear equations arising from integral-equation formulations," IEEE Antennas Propag. Mag, vol. 37, pp. 96-100, 1995.

[5] J. Song, C. C. Lu, and W. C. Chew, "Multilevel fast multipole algorithm for electromagnetic scattering by large complex objects," IEEE Trans. Antennas Propag., vol. 45, pp. 1488-1493, 1997.

[6] K. Sertel and J. L. Volakis, "Incomplete LU preconditioning for FMM implementation," Microw. Opt. Technol. Lett., vol. 26, no. 4, Aug. 2000.

[7] J. Lee, J. Zhang, and C-C. Lu, "Incomplete LU preconditioning for large scale dense complex linear systems from electromagnetic wave scattering problems," J. Comput. Phys., vol. 185, pp. 158-175, Feb. 2003.

[8] T. F. Eibert, "Iterative near-zone preconditioning of iterative method of moments electric field equation solutions," IEEE Antennas Wireless Propag. Lett., vol. 2, pp. 101-102, 2003.

[9] M. Carr, M. Bleszynski, and J. L. Volakis, "A near-field preconditioner and its performance in conjunction with the BiCGsatbn(ell) solver," IEEE Antennas Propag. Mag., vol. 46, no. 2, pp. 23-30, Apr. 2004.

[10] J. R. Poirier, P. Borderies, R. Mittra, and V. Varadarajan, "Numerically efficient solution of dense linear system of equations arising in a class of electromagnetic scattering problems," IEEE Trans. Antennas Propag., vol. 46, no. 8, pp. 1169-1175, Aug. 1998.

[11] R. A. Shelby, D. R. Smith, S. C. Nemat-Nasser, and S. Schultz, "Microwave transmission through a two-dimensional, isotropic, left-handed metamaterial," Appl. Phys. Lett., vol. 78, no. 4, 2001.

[12] E. Ubeda, A. Heldring, J. Parron, J. Romeu, and J. Rius, "Radiation pattern of composite finite arrays of conduting elements with an exciting elementary dipole," presented at the IEEE AP-Symp., Columbus, $\mathrm{OH}$, Jun. 22-27, 2003.

[13] S. M. Rao, D. R. Wilton, and A. W. Glisson, "Electromagnetic Scattering by Surfaces of Arbitrary Shape," IEEE Trans. Antennas Propag., vol. AP-30, no. 3, pp. 409-418, May 1982.

[14] D. R. Wilton, A. W. Glisson, D. H. Schaubert, O. M. Al-Bundak, and C. M. Butler, "Potential Integrals for uniform and linear source distributions on polygonal and polyhedral domains," IEEE Trans. Antennas Propag., vol. AP-32, no. 3, pp. 276-281, Mar. 1984.

[15] E. Ubeda and J. M. Rius, "Análisis eficiente de Slabs de Metamateriales," in Simposium Nacional de la Unión Científica internacional de Radio, Barcelona, Spain, Sep. 8-10, 2004.

[16] A. Heldring, J. M. Rius, L. P. Ligthart, and A. Cardama, "Accurate numerical modeling of the TARA reflector system," IEEE Trans. Antennas Propag., vol. 52, no. 7, pp. 1758-1766, Jul. 2004.

\section{Physical Insight Into the "Growing” Evanescent Fields of Double-Negative Metamaterial Lenses Using Their Circuit Equivalence}

\author{
Andrea Alù and Nader Engheta
}

Abstract-Pendry in his paper, "Negative refraction makes a perfect lens" (Phys. Rev. Lett., vol. 85, no. 18, pp. 3966-3969, 2000) put forward an idea for a lens made of a lossless metamaterial slab with $n=-1$, that may provide focusing with resolution beyond the conventional limit. In his analysis, the evanescent wave inside such a lossless double-negative (DNG) slab is "growing," and thus it "compensates" the decaying exponential outside of it, providing the subwavelength lensing properties of this system. Here, we examine this debated issue of "growing exponential" from an equivalent circuit viewpoint by analyzing a set of distributed-circuit elements representing evanescent wave interaction with a lossless slab of DNG medium. Our analysis shows that, under certain conditions, the current in series elements and the voltage at the element nodes may attain the dominant increasing due to the suitable resonance of the lossless circuit, providing an alternative physical explanation for "growing exponential" in Pendry's lens and similar subwavelength imaging systems.

Index Terms-Double-negative (DNG) metamaterials, left-handed (LH) metamaterials, subwavelength resolution.

\section{INTRODUCTION}

The idea of left-handed (LH) media, which dates back to 1967 when Veselago [1], theoretically studied plane wave propagation in materials in which he assumed both permittivity and permeability simultaneously having negative real parts, has attracted a great deal of attention in recent years. Various problems and ideas involving such media have been proposed and studied by many research groups. One such idea, namely a lens with possibility of perfect focusing, was theoretically suggested by Pendry in [2]. In his analysis, Pendry shows how evanescent waves, which are effectively responsible for subwavelength resolution, impinging on a suitably designed slab of double-negative (DNG) [3] material, may grow exponentially inside such a slab, and how this effect may "compensate" the decaying exponential taking place outside the slab [2]. This issue of "growing exponential" and subwavelength imaging has become the subject of interest for several research groups working in metamaterial research (see, e.g., [4]-[7]). Analogous subwavelength focusing and growing evanescent distributions have been demonstrated in two-dimensional negative-refractive-index transmission line structures [8], [9].

In one of our previous works, we have shown how a similar phenomenon of "growing exponential" may occur in pairs of "conjugate" metamaterial slabs, i.e., pairs of DNG and double-positive (DPS) slabs or pairs of single-negative (SNG) layers such as epsilon-negative (ENG) and $\mu$-negative (MNG) layers [10]. In these cases, we have shown how wave tunneling, transparency, and virtual image subwavelength displacement may be achieved under a proper choice of combinations

Manuscript received August 18, 2004; revised October 3, 2005. This work was supported in part by the Fields and Waves Laboratory, Department of Electrical and Systems Engineering, University of Pennsylvania. The work of A. Alù was supported by the scholarship "Isabella Sassi Bonadonna" from the Italian Electrical Association (AEI).

A. Alù is with the Department of Applied Electronics, University of Roma Tre, Rome, Italy and also with the Department of Electrical and Systems Engineering, University of Pennsylvania, Philadelphia, PA 19104 USA (e-mail: alu@uniroma3.it; andreaal@ee.upenn.edu).

N. Engheta is with the Department of Electrical and Systems Engineering, University of Pennsylvania, Philadelphia, PA 19104 USA (e-mail: engheta@ ee.upenn.edu.

Digital Object Identifier 10.1109/TAP.2005.861509 


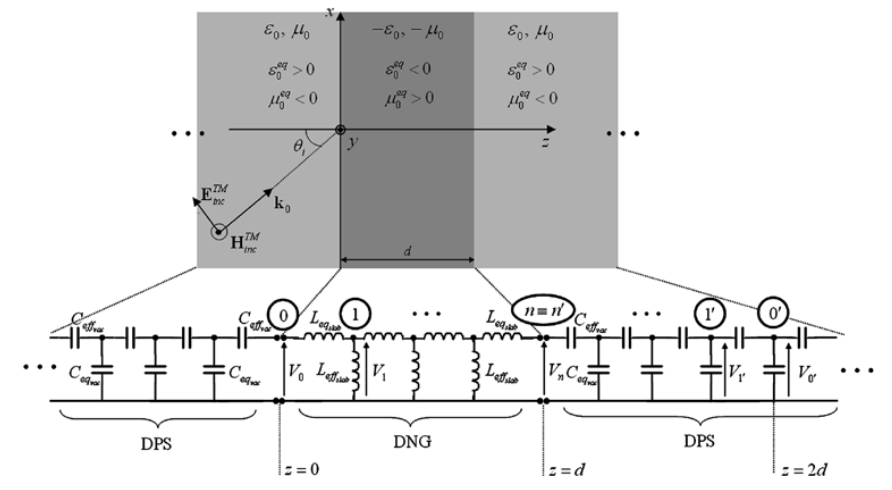

Fig. 1. Geometry of the problem and equivalent TL model for a TM evanescent wave impinging on the Pendry's perfect lens.

of metamaterial parameters and slab thicknesses, independent on the property of the "outside" medium that surrounds the pairs of slabs. We attributed these findings to the presence of an "interface resonance" at the boundary between the two conjugate slabs. It is worth noting that these pairs of conjugate slabs also supported growing evanescent fields internally when an incident wave impinges on them. We showed how the "interface resonance" may be explained using the circuit-element analogy, when the conjugate distributed-circuit elements are paired to produce similar resonances and growing distributions for the voltage and currents.

In the present communication, we apply the same concepts to explain how Pendry's lens may be viewed as a special case of a more general analysis of pairs of conjugate slabs described in our previous work [10] and how the growing evanescent field behavior in his lens may be clearly understood using the equivalent circuit analogy. Some of our preliminary results in this work were presented in a recent symposium [11].

\section{Formulation OF THE PROBlem}

As in Pendry's lens case [2], here we consider a plane wave impinging on a metamaterial slab with $\varepsilon_{\text {slab }} / \varepsilon_{0}=\mu_{\text {slab }} / \mu_{0}=-1$, as depicted in Fig. 1 (top). The electromagnetic problem can be solved by matching the boundary conditions at the interfaces $z=0$ and $z=d$, obtaining the values for the Fresnel transmission and reflection coefficients. The solution implies that on the plane $z=2 d$ each plane wave, whether propagating or evanescent, would have exactly the same value as the one it has at $z=0$, essentially showing how such an ideal "matched" DNG slab acts as a "perfect" lens, providing an image of the object plane at $z=0$ without any limit on the resolution [2]. The evanescent waves inside this DNG slab, moreover, "grow" instead of decaying for both polarizations. As we show in the following, an equivalent circuit representation may provide a similar, but arguably more familiar, behavior for voltages and currents in suitably selected distributed circuit elements, thus providing new physical insights into this phenomenon.

\section{Circuit EQuivalence}

Consistently with our analysis in [10], the $e^{-j k_{x} x}$ plane wave propagation in a homogeneous isotropic medium with permittivity $\varepsilon$ and permeability $\mu$ may be viewed as formally analogous to the transmission line equations $\partial V / \partial z=-j \omega L_{\mathrm{eq}} I, \partial I / \partial z=-j \omega C_{\mathrm{eq}} V$, with the equivalent series inductance per unit length $L_{\mathrm{eq}}$ and equivalent shunt capacitance per unit length $C_{\text {eq }}$ being proportional respectively to the equivalent permeability and permittivity, which in the TM case are given by $\tilde{\mu}_{\mathrm{eq}} \equiv \mu\left[1-k_{x}^{2} /\left(\omega^{2} \mu \varepsilon\right)\right], \tilde{\varepsilon}_{\mathrm{eq}} \equiv \varepsilon$ (the TE case may be easily obtained by duality). It is worth noting that the transmission-line analogy may in general offer an interesting physical interpretation and alternative insight, effectively linking the voltage and current distributions along a circuit network to their local counterparts represented by the electric and magnetic fields. This is well known in the DPS case [12], but it is easily extended to the metamaterial parameters, as it has been shown in [8]-[10].

We note that even in a conventional DPS material, where $\mu$ and $\varepsilon$ are positive, the value of $L_{\mathrm{eq}}$ in the TM case and $C_{\mathrm{eq}}$ in the TE case may become negative, when $k_{x}^{2}>\omega^{2} \mu \varepsilon$, i.e., for an evanescent wave. As is well known, a negative equivalent inductance or capacitance at a given frequency may be interpreted effectively as a positive (dispersive) capacitance or inductance at that frequency, respectively [8], [10]. Therefore, for the TM case the evanescent plane wave propagation in a DPS medium may be modeled using a transmission line with a negative series inductance per unit length and a positive shunt capacitance per unit length, which effectively implies a positive series capacitance per unit length and a positive shunt capacitance per unit length. In such a $C-C$ line, which is a ladder network made of capacitors, currents and voltages cannot "propagate" along the line, but instead they have an evanescent behavior, consistently with the electromagnetic counterpart. When a DNG material or an ENG or MNG medium is used, their suitable equivalent TL models may exhibit anomalous properties consistent with the features of wave propagation in such media. For the TE polarization, one may consider Table I showing the equivalent TL model for plane waves in lossless homogeneous isotropic media, with all possibilities for signs of the real part of their permittivity and permeability, both for the cases of propagating and evanescent waves. When losses are present, $\mu$ and/or $\varepsilon$ have complex values, which translates into positive series resistance and/or shunt conductance in the TL model. (A similar table for the TM polarization was presented in [10].)

If we now consider Pendry's lens problem, the equivalent 1-D TL model may be depicted as in Fig. 1 (bottom), where a TM evanescent wave, impinging on a "matched" DNG slab, is considered. In the figure, we have considered $k_{x}^{2}>\omega^{2} \mu_{0} \varepsilon_{0}=\omega^{2} \mu_{\text {slab }} \varepsilon_{\text {slab }}$, which gives an evanescent wave in the vacuum and inside the slab. The primary parameters of the TL sections may be derived from the previous discussion: when the equivalent inductors or capacitors are negative, in the figure they are respectively shown as effective capacitors or inductors, i.e., since $C_{\mathrm{eq}_{\mathrm{slab}}}<0$ and $L_{\mathrm{eq}} \mathrm{vac}, 0$, we have $\left(j \omega C_{\mathrm{eq}_{\mathrm{slab}}}\right)^{-1}=j \omega L_{\mathrm{eff}_{\mathrm{slab}}}$, and $j \omega L_{\mathrm{eq}_{\mathrm{vac}}}=\left(j \omega C_{\mathrm{eff}_{\mathrm{vac}}}\right)^{-1}$. Moreover, since for Pendry's "matched" DNG slab $C_{\mathrm{eq}_{\mathrm{slab}}}=-C_{\mathrm{eq}}$ and $L_{\mathrm{eq}}=-L_{\mathrm{eq}} \mathrm{q}_{\mathrm{slab}}$ for any $k_{x}$, their values satisfy the following relations:

$$
L_{\mathrm{eq}_{\mathrm{slab}}} C_{\mathrm{eff}}=L_{\mathrm{vac}}{ }_{\mathrm{slab}} C_{\mathrm{eq}_{\mathrm{vac}}}=\omega^{-2} \quad \forall k_{x} .
$$

From this relation, we get the following expressions for the secondary parameters $Z$ and $\kappa$ of each line segment, shown in (2) at the bottom of the page, which ensure that the magnitudes of the characteristic impedances and of the wave numbers are the same in the two lines,

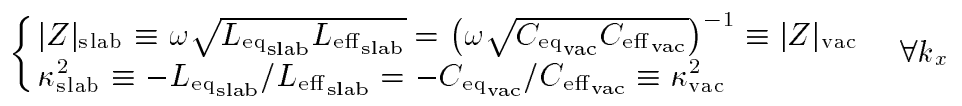


TABLE I

EFFective TL Models in Lossless DPS, DNG, ENG, MNG SLABS FOR THE TE PROPAGATING AS WELL AS EvANESCENT WAVES

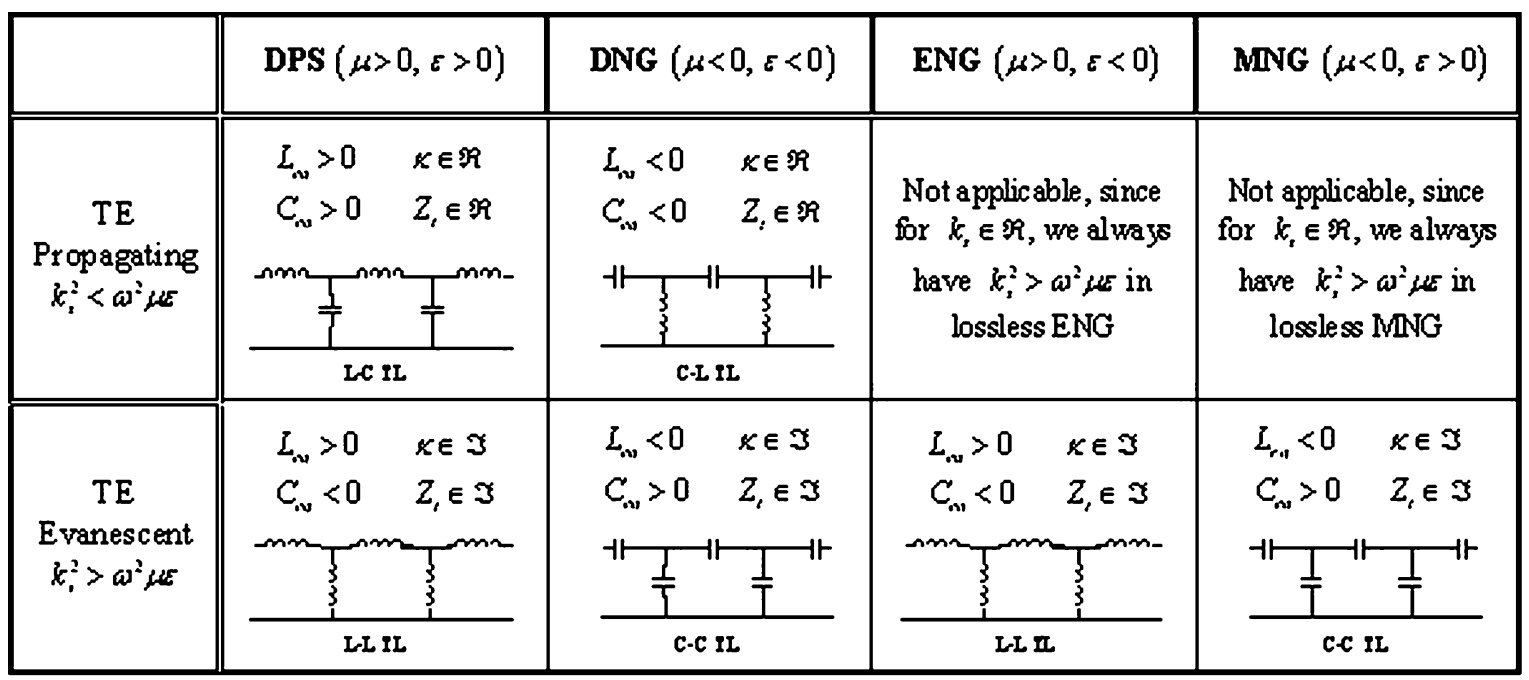

and that they are all imaginary quantities (since the wave is evanescent). Nothing is said in (2) on their signs, but they may be derived from the following considerations.

The signs of the imaginary wave numbers $\kappa$ in the $C-C$ and $L-L$ ladders have to be negative, to ensure the exponential causal decay in an infinite or a matched line with $e^{-j \kappa z}$ propagating factor. For what concerns the signs of the characteristic impedances for an $L-L$ or $C-C$ line, we obviously expect to have inductive or capacitive characteristic impedance for these lines, respectively. (A more rigorous demonstration may be obtained by adding a small amount of losses to the TL parameters, in order to select the proper branches of the square roots with positive real part, similarly to what shown in [3].) This results in the following expressions (where we choose always the positive sign for the square roots):

$$
\begin{aligned}
& \kappa_{\mathrm{vac}}=-j \sqrt{C_{\mathrm{eq}_{\mathrm{vac}}} / C_{\mathrm{eff}_{\mathrm{vac}}}}, \kappa_{\mathrm{slab}}=-j \sqrt{L_{\mathrm{eq}_{\mathrm{slab}}} / L_{\mathrm{eff}_{\mathrm{slab}}}} \\
& Z_{\mathrm{vac}}=\left(j \omega \sqrt{C_{\mathrm{eq}} C_{\mathrm{vac}} C_{\mathrm{eff}_{\mathrm{vac}}}}\right)^{-1}, Z_{\mathrm{slab}}=j \omega \sqrt{L_{\mathrm{eq}_{\mathrm{slab}}} L_{\mathrm{eff}} \mathrm{slab}} .
\end{aligned}
$$

These formulas clearly show that, unlike the case of propagating wave interaction with this DNG slab where the impedances are matched [2], [3], for the evanescent waves the two media are not impedance-matched, since $Z_{\mathrm{vac}}=-Z_{\mathrm{slab}}$, but on the other hand at the interface a resonance arises, giving rise to a reflection coefficient $R=Z_{\mathrm{vac}}-Z_{\text {slab }} / Z_{\mathrm{vac}}+Z_{\text {slab }}=\infty$. This "interface" resonance is the key in understanding the anomalous behavior of this setup, and the circuit analogy gives a further insight into this phenomenon.

Equation (2) and the previous consideration, in fact, imply that at each of the two interfaces between vacuum and the DNG slab, the adjacent series elements $C_{\mathrm{eff}}$ vac and $L_{\mathrm{eq}_{\mathrm{slab}}}$ would resonate at the frequency of operation for any $k_{x}$ (this is of course the circuital counterpart of the surface waves supported by such an interface [2], [13], which indeed play a key role in the physics of the subwavelength imaging [5]-[7]). Therefore, looking at the right interface (node $n=n^{\prime}$ in the figure), we note that the voltage at the left node of this $L_{\text {eq }}$ slab $(n-1$ in the figure) is the same as the voltage at the right node of this $C_{\text {eff }}$ vac ( $n^{\prime}-1$ in the figure). Consequently, the next two adjacent shunt elements $L_{\text {eff }}$ slab and $C_{\mathrm{eq}_{\mathrm{vac}}}$ are now in parallel and they are also in resonance, again according to (2). Repeating this argument, we note that effectively a segment of the $C-C$ line with length $d$ would be in resonance with the entire $L-L$ line that represents the matched DNG slab with the same thickness $d$. In fact, we expect that the voltage and current at every node $i$ are the same as those at the corresponding node $i^{\prime}$. Therefore, the voltage and current at one end (node 0 ) of this "resonant pair" of $C-C$ and $L-L$ lines (each with length $d$ ) would be the same at those at its other end (node 0 '), which implies that this pair appears to become "transparent" to the rest of the structure. This also means that if in the $C-C$ segment we have a decaying exponential voltage (which is the only physical possibility), we should have a "growing exponential" voltage in the $L-L$ segment in order to have the voltage nodes the same at the beginning and at the end of this pair. In fact, due to the multiple reflections at the two interfaces, each with an "infinite" reflection coefficient, the "reflected growing" exponential builds up in the steady-state regime and totally dominates the impinging decaying exponential in the DNG slab by itself. It is important to underline here that the presence of the "growing" exponential in the $L-L$ line is due to the "interface" resonance at the boundary between the $C-C$ and $L-L$ lines, and it is not just only due to the $L-L$ line (i.e., the DNG slab) by itself [as confirmed also by (3)]. In other words, in a dual scenario if we had had a "vacuum" slab sandwiched between two semi-infinite DNG half spaces, following a similar argument we would have seen the growing exponential in the vacuum slab region!

A further confirmation of the presence of the growing exponential in the $L-L$ line segment sandwiched as in Fig. 2 may be found directly by solving such a circuit network. Let us excite this circuit with a steadystate time-harmonic voltage source $V_{\text {exc }}$ at a given node in the semiinfinite $C-C$ transmission line on the left of the $L-L$ segment. We have shown above that the pair of $L-L$ segment together with the $d$ -long $C-C$ segment is in resonance and thus "transparent" to the rest of the structure. Therefore, we expect to have the voltage and current in the left $C-C$ segment to be related as follows:

$$
I_{\mathrm{exc}}=\frac{V_{\mathrm{exc}}}{Z_{\mathrm{vac}}}=j \omega \sqrt{C_{\mathrm{eq}} C_{\mathrm{vac}} C_{\mathrm{eff}}} V_{\mathrm{exc}}=\frac{j V_{\mathrm{exc}}}{\omega \sqrt{L_{\mathrm{eq}_{\mathrm{slab}} L_{\mathrm{eff}} \mathrm{slab}}}}
$$

as in any matched or infinite line. For the same reason, the node voltages and branch currents along the $C-C$ line in the left decay exponentially, due to the imaginary value of $\kappa_{\mathrm{vac}}$ shown in (3), until we get to the left interface between the $C-C$ and $L-L$ lines, which is denoted in the figure with node " 0 ". Let us denote the node voltage at this interface $V_{0}$, and the series branch current $I_{0}$, which can be expressed as $I_{0}=j \omega \sqrt{C_{\mathrm{eq}} C_{\mathrm{vac}} C_{\mathrm{eff}}{ }_{\mathrm{vac}}} V_{0}$, following (4). As we move into 
the $C-C$ line, we can evaluate the node voltage $V_{n}$ and the current $I_{n}$ in the following branch at the $n$th node with the recursive relations:

$$
\begin{aligned}
V(n) & =V(n-1)-j \omega L_{\mathrm{eq}_{\mathrm{slab}}} I(n-1) \\
I(n) & =I(n-1)-V(n) /\left(j \omega L_{\mathrm{eff}_{\mathrm{slab}}}\right) \\
V(0) & =V_{0} \\
I(0) & =j \omega \sqrt{C_{\mathrm{eq}_{\mathrm{vac}} C_{\mathrm{eff}}{ }_{\mathrm{vac}}}} V_{0} \\
& =\left(-j \omega \sqrt{L_{\mathrm{eq}_{\mathrm{slab}}} L_{\mathrm{eff}_{\mathrm{slab}}}}\right)^{-1} V_{0}=I_{0} .
\end{aligned}
$$

This is analogous to the Fibonacci problem, and the explicit solution may be written for even $n$ as

$$
\begin{aligned}
V(n)= & \frac{V_{0}(4 \gamma+1)^{n / 2-1}}{(2 \gamma)^{n-1}} \\
& \cdot \sum_{k=0}^{n-2} \text { even }\left(\begin{array}{c}
n-1 \\
k
\end{array}\right) \\
& \times\left[\frac{n-k-1}{k+1}(2 \gamma+1)+2 \sqrt{\gamma}-1\right]\left(\frac{2 \gamma+1}{\sqrt{4 \gamma+1}}\right)^{k} \\
I(n)= & \frac{I_{0}(4 \gamma+1)^{n / 2-1}}{(2 \gamma)^{n-1}} . \\
& \cdot \sum_{k=0}^{n-2} \text { even }\left(\begin{array}{c}
n-1 \\
k
\end{array}\right) \\
& \times\left[\frac{n-k-1}{k+1}(2 \gamma+1)+2 \sqrt{\gamma}+1\right]\left(\frac{2 \gamma+1}{\sqrt{4 \gamma+1}}\right)^{k}
\end{aligned}
$$

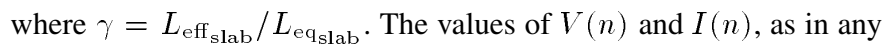
pseudo-Fibonacci series, grow exponentially with $n$. This growth continues until we reach the right interface of the $L-L$ segment. Beyond this interface, we are in the $C-C$ segment, and with a similar argument we expect to have a decaying exponential with symmetrical values as in the $L-L$ segment, i.e., $V\left(j^{\prime}\right)=V(j), I\left(j^{\prime}\right)=I(j)$ for any $0 \leq j \leq n$. Therefore, the maximum values of $V(n)$ and $I(n)$ are expected to be at the interface $n=n^{\prime}$, as predicted by Pendry in his DNG slab [2]. We have thus far shown how a "growing" exponential behavior inside a matched DNG slab of thickness $d$ may be justified using the circuit equivalence with the $L-L$ and $C-C$ lines. In fact, as we have mentioned in our previous work, one can suggest that this field behavior may also exist when a DNG slab is juxtaposed with a "conjugate" DPS slab of the same thickness (in the present case, this DPS layer is part of the outside vacuum region), or an epsilon-negative (ENG) slab paired with a mu-negative (MNG) slab of equal thickness [10] (and these cases would show total transparency independently of the parameters of the outside region surrounding the system).

In principle, this anomalous transparency is independent of the thickness of the two slabs (or in Pendry's lens of the DNG slab itself), as long as the slabs have equal thickness $d$. However, an important question may arise here: May we still have a growing exponential behavior inside the DNG slab (or equivalently inside the $L-L$ line here), if its thickness becomes infinite, i.e., if we have a semi-infinite DNG space? According to the analysis presented here, the answer is as follows: we need both interfaces to achieve this exponential growth, therefore this effect may not take place if the second interface is at infinity. Moreover, due to the multiple resonances/reflections that are necessary for the phenomenon to build up, a thicker slab should be more sensitive to the inherent losses of the setup and more time should be also required for the phenomenon to build up and reach the steady-state regime. Therefore, even if the second interface is too far apart (and not at infinity) in practical systems the growing exponential may disappear. This is consistent with the findings reported in the literature (see, e.g., [14], [15]).
It is known, however, that an interface between the semi-infinite matched DNG and DPS media may indeed support a surface plasmon wave [2], [5], [13]. In this case, for an incoming evanescent wave, the transverse impedances of the two regions are complex conjugate of each other, i.e., $Z_{L-L} \equiv-Z_{C-C}=j X$, and therefore the Fresnel "reflection" and "transmission" coefficients for such an incident evanescent wave become infinite, as we have previously found for each of the two interfaces in the circuit analog. We reiterate that this in principle does not violate any physical law, since these coefficients here describe the relation between an "incident" evanescent wave and its "reflected" and "transmitted" evanescent waves, neither of which carries any real power. So when we have a source in front of the interface between two semi-infinite matched DNG and DPS media, the resonant surface wave may be excited along the interface, resulting in an infinitely large field value. However, on both sides of this interface, the fields, albeit infinitely large, decay exponentially, since the field distribution represents a surface wave propagating along such an interface.

\section{EFFECTS OF LOSS AND MISMATCH IN MATERIALS}

Thus far we have assumed complete losslessness and match between the DNG and the outside region. When loss and/or mismatch in the material parameters is present, we expect to have certain variations to the field distribution in this geometry, and as the DNG slab gets thicker, such variations would be more sensitive to the presence of loss and mismatch, as also anticipated. This sensitivity is mainly due to the presence of the surface wave supported by the slab (notice that the matched slab without losses, in fact, does not support any surface wave, even thought the two interfaces delimiting the slab would do so). If the structure supports a surface wave with a given $k_{x}>k_{0}$, in fact, the reflection and transmission coefficients for the DNG slab would no longer be flat for all $k_{x}$, but instead would experience a peak (or a singularity in case of no loss) at the value of $k_{x}$ for which the surface wave is supported. This has been shown by others in several recent papers studying this phenomenon [16]-[18] and indeed limits the overall resolution to certain extent, which can still be subwavelength, better than the conventional resolution.

The effects of loss and mismatch may again be explained by the equivalent circuit models described here. In the ideal lossless matched case, we showed that for any value of $k_{x}$, the $C_{\text {eff }}$ vac series capacitors are all in resonance with the corresponding series $L_{\mathrm{eq}}$ slab and similarly every $C_{\mathrm{eq}}$ sac shunt capacitor is in resonance with a corresponding shunt $L_{\text {eff }}$ slab. The quality factor $Q$, of such a resonance is thus infinitely large. However, the loss in the system causes the quality factor $Q$ to become finite, resulting in a quicker drop of the transmission for high $k_{x}$ (for which the equivalent electrical length of the TL increases). Moreover, the mismatch does not allow a "perfect" resonance between the inductors and capacitors mentioned above for all value of $k_{x}$ : only for certain $k_{x}$ such a resonance may still occur.

\section{CONCLUSION}

Considering the plane wave interaction with Pendry's "perfect" lens, we have shown how this problem may be treated equivalently as a finite segment of $L-L$ line, representing the DNG slab for the evanescent wave, sandwiched between two semi-infinite segments of $C-C$ lines, representing the outside DPS regions for the TM evanescent wave. In this analogy, voltages and currents represent the electric and magnetic fields. We have analyzed the overall circuit, showing the possibility of explaining the growing exponential term for the electromagnetic field along the DNG segment as a resonant phenomenon in the circuit, with an analogous growth of voltage and current distributions. The model is effective also in presence of losses, 
which are represented by resistances and conductances and may give further insights into the anomalous phenomenon of subwavelength imaging utilizing metamaterials.

\section{REFERENCES}

[1] V. G. Veselago, "The electrodynamics of substances with simultaneously negative values of $\varepsilon$ and $\mu$,' Soviet Phys. Uspekhi, vol. 10, no. 4, pp. 509-514, 1968.

[2] J. B. Pendry, "Negative refraction makes a perfect lens," Phys. Rev. Lett., vol. 85, no. 18, pp. 3966-3969, 2000.

[3] R. W. Ziolkowski and E. Heyman, "Wave propagation in media having negative permittivity and permeability," Phys. Rev. E, vol. 64, no. 5, p. 056625, Oct. 30, 2001.

[4] S. Maslovski and S. Tretyakov, "Phase conjugation and perfect lensing," J. Appl. Phys., vol. 94, no. 7, pp. 4241-4243, 2003.

[5] M. W. Feise, P. J. Bevelacqua, and J. B. Schneider, "Effects of surface waves on the behavior of perfect lenses," Phys. Rev. B, vol. 66, p. 035113 , July 22, 2002.

[6] C. Luo, S. G. Johnson, J. D. Joannopoulos, and J. B. Pendry, "Subwavelength imaging in photonic crystals," Phys. Rev. B, vol. 68, p. 045115 , Jul. 29, 2003.

[7] D. R. Smith, D. Schurig, and J. B. Pendry, "Negative refraction of modulated electromagnetic waves," Appl. Phys. Lett., vol. 81, no. 15, pp. 2713-2715, Oct. 7, 2002.

[8] G. V. Eleftheriades, A. K. Iyer, and P. C. Kremer, "Planar negative refractive index media using periodically L-C loaded transmission lines," IEEE Trans. Microw. Theory Tech., vol. 50, no. 10, pp. 2702-2717, Dec. 2002.

[9] A. Grbic and G. V. Eleftheriades, "Growing evanescent waves in negative-refractive-index transmission-line media," Appl. Phys. Lett., vol. 82, no. 12, pp. 1815-1817, Mar. 24, 2003.

[10] A. Alù and N. Engheta, "Pairing an epsilon-negative slab with a mu-negative slab: Anomalous tunneling and transparency," IEEE Trans. Antennas Propag., Special Issue on Metamaterials, vol. AP-51, no. 10, pp. 2558-2570, Oct. 2003.

[11] - "Circuit equivalence of "growing exponential" in Pendry's lens," in Proc. USNC/CNC/URSI Nat. Radio Science Meet., Columbus, OH, Jun. 22-27, 2003, p. 22.

[12] R. E. Collin, Field Theory of Guided Waves. New York: IEEE Press, 1991.

[13] A. Alù and N. Engheta, "Radiation from a traveling-wave current sheet at the interface between a conventional material and a metamaterial with negative permittivity and permeability," Microw. Opt. Technol. Lett., vol. 35, no. 6, pp. 460-463, Dec. 20, 2002.

[14] A. Alù, N. Engheta, and R. W. Ziolkowski, "FDTD simulation of tunneling and "growing exponential" in a pair of $\varepsilon$-negative and $\mu$-negatives slabs," in Proc.IEEE AP-S Int. Symp. and USNC/CNC/URSI Nat. Radio Science Meet. Dig., Monterey, CA, Jun. 20-26, 2004, p. 18.

[15] S. A. Tretyakov, Analytical Modeling in Applied Electromagnetics. New York: Artech House, 2003.

[16] A. Ishimaru and J. R. Thomas, "Transmission and focusing properties of a slab of negative refractive index," in Proc. USNC/URSI Nat. Radio Science Meeting, San Antonio, TX, 2002, p. 43.

[17] N. Fang and X. Zhang, "Imaging properties of a metamaterial superlens," Appl. Phys. Lett., vol. 82, no. 2, pp. 161-163, Jan. 13, 2003.

[18] D. R. Smith, D. Schurig, M. Rosenbluth, S. Schultz, S. A. Ramakrishna, and J. B. Pendry, "Limitations on subdiffraction imaging with a negative refractive index slab," Appl. Phys. Lett., vol. 82, no. 10, pp. 1506-1508, Mar. 10, 2003.

\section{Backscattering from Square Plates Illuminated With Vertical Polarization}

\author{
R. Alexander Ross
}

Abstract-The geometrical theory of diffraction is used to describe backscattering by a square flat plate as a function of aspect angle. When the plate is illuminated with vertical polarization, singly diffracted terms are augmented by a new doubly diffracted contribution that accounts for grazing phenomena. The latter term is obtained from a related analysis that describes bistatic scattering from a flat plate. Predictions are compared with numerical results from the method of moments for plates between 2 and 7 wavelengths on a side. The modified theory accurately predicts the radar cross section of square plates as small as 3 wavelengths on a side.

Index Terms-Geometrical theory of diffraction (GTD), grazing incidence, radar cross section (RCS), square flat plates.

\section{INTRODUCTION}

Electromagnetic scattering from flat surfaces has been treated extensively by Keller's geometrical theory of diffraction (GTD) [1] and by the uniform theory of diffraction (UTD) [2]. Ross [3] applies GTD with multiple diffraction to predict backscattering from rectangular flat plates. Tiberio and Kouyoumjian [4] use UTD to analyze grazing incidence on strips. Polka et al. [5] compare UTD predictions with measurements for narrow rectangular plates and present measurements of the oscillation in radar cross section (RCS) with frequency at grazing incidence. Pelosi et al. [6] examine a square plate to point out difficulties in applying GTD. Sitka et al. [7] include a first-order comparison of the modified equivalent current (MEC) and corner diffraction calculations of backscattering from a small square plate. Senior [8] discusses grazing phenomena in an investigation of disk scattering at edge-on incidence. Beyond determinate analyzes, Hestilow [9] reported a statistical analysis of the average RCS of cylinders and rectangular plates over a symmetric window around broadside. These studies do not remedy the failure of aysmptotic theory to account for grazing phenomena observed with finite rectangular plates at least several wavelengths on a side. Both backscattering and forward scattering can exhibit large effects when a plate is illuminated near edge-on incidence with vertical polarization.

The topic of greatest interest in this paper is monostatic scattering from square plates. However, bistatic scattering is used as part of the monostatic solution. Briefly, grazing phenomena are interpreted as the bistatic contribution that is doubly diffracted at the rear edge of the plate. Formulas are derived using GTD and include an empirical term. Accuracy is demonstrated by comparing predictions with calculations based upon the method of moments (MoM).

\section{ANALYSIS}

Fig. 1 shows a plate lying in the $Y-Z$ plane of the coordinate system used for analysis. The square plate is $2 a$ on a side. It is assumed to be vanishingly thin compared to a wavelength, and perfectly conducting. Illumination by a plane wave is depicted with the wave propagation vector confined to the $X-Y$ plane. The monostatic aspect angle $\alpha$ is measured from the $X$-axis and the phase reference is the origin. Our investigation is restricted to vertical polarization: in Fig. 1, the $E$ vector is parallel to the $Z$-axis. The size of the plates varies from $2 \lambda$ to $7 \lambda$,

Manuscript received March 26, 2004; revised June 9, 2005.

The author is with Integrated Defense Systems (IDS), Raytheon, Bedford, MA 01730 USA (e-mail: alexross@11.mit.edu).

Digital Object Identifier 10.1109/TAP.2005.861506 